This is the post print version of the article, which has been published in Journal of child and adolescent psychopharmacology . 2019, 29 (1), 72-74. https:// doi.org/10.1089/cap.2018.0084.

\title{
Tardive dyskinesia should not be overlooked
}

Kakko Kirsi, MD. University of Tampere, Faculty of Medicine and Life Sciences, Tampere, Finland. Tampere University Hospital, Department of Child Psychiatry, Tampere, Finland

Bjelogrlic-Laakso Nina, MD, PhD. Tampere University Hospital, Intellectual Disability Services, Tampere, Finland

Pihlakoski Leena, MD. Tampere University Hospital, Department of Child Psychiatry, Tampere, Finland

Lehtimäki Kai, MD, PhD. Tampere University Hospital, Department of Neurosciences, Neurology and Rehabilitation, Tampere, Finland. University of Tampere, Faculty of Medicine and Life Sciences, Tampere, Finland

Järventausta Kaija, MD, PhD. Tampere University Hospital, Department of Psychiatry, Tampere, Finland. University of Tampere, Faculty of Medicine and Biosciences, Tampere, Finland

Corresponding author:

Kirsi Kakko, MD.

University of Tampere, Faculty of Medicine and Life Sciences, Tampere, Finland.

Tampere University Hospital Department of Child Psychiatry

PL 2000, 33521 Tampere, Finland.

kirsi.kakko@uta.fi

$+358405779250$

\section{Acknowledgments}

This study was supported by the Foundation for Pediatric Research and the Finnish Brain Foundation. 


\section{Introduction}

Medicating the comorbid behavioral symptoms of children and adolescents with autism spectrum disorders (ASD) and intellectual disability (ID) with second-generation antipsychotics (SGA) has become accepted practice in recent years (Park et al. 2016). Although the available research data covers at most short-term use, SGAs are often used for long periods in clinical settings. There are also reports of electroconvulsive therapy (ECT) in treating aggressive and self-injurious behaviors in pediatric patients with ASD/ID, but as current evidence is based on case reports, ECT is an off-label treatment method (Wachtel et al. 2018, Sajith et al. 2017).

While SGAs are generally considered more tolerable than first-generation antipsychotics, they can still increase the risk for extrapyramidal symptoms (EPS) (Garcia-Amador et al. 2015). EPS rates in children and adolescents vary in studies (Carbon et al. 2015, Garcia-Amador et al. 2015, Pringsheim et al. 2017), but a younger age, a longer duration of treatment, and higher doses associate with more severe manifestations, such as tardive dyskinesia (TD) (Garcia-Amador et al. 2015). Patients with ID are more susceptible to EPS (Sheehan et al. 2017).

TD is probably the most feared and potentially irreversible form of EPS. TD manifests usually after months or years of medication as involuntary athetoid or choreiform movements and dystonia, often affecting the facial area and tongue (Lerner et al. 2015). Treatment options for TD include pharmacological interventions (Lerner et al. 2015), but severe TD is often permanent. There is encouraging evidence for deep brain stimulation (DBS) in treating severe, medication-resistant TD in adults (Macerollo and Deuschl 2018), and also in the treatment of dystonic cerebral palsy in children (Elia et al. 2018). To our knowledge, however, this is the first case reports (Macerollo and Deuschl 2018) describing DBS in the treatment of TD in an adolescent patient. 


\section{Case example}

Our patient is an adolescent male with a diagnosis of ASD and ID (ICD-10 diagnosis F71, moderate mental retardation). He communicates using, for example, pictures, and he understands short sentences. Risperidone ( $0.25 \mathrm{mg}$ daily) was first initiated - with a favorable response - for aggressive behavior and tantrums when he was 8 years old. During the first two years, the daily dose of risperidone was increased to $0.4 \mathrm{mg}$. Higher dosages appeared to cause tics and sedation. Despite the medication, the patient's aggression and behavioral symptoms further worsened between the ages 13 and 16, and the risperidone dose was gradually increased to $3 \mathrm{mg}$ daily. Melatonin (1.5 mg daily) and later chlorprothixene (thioxanthene; $25 \mathrm{mg}$ daily) were prescribed for transient sleep problems. Regardless of pharmacotherapy, his behavioral symptoms continued and worsened. Just before the patient turned 16 he became constantly restless, his aggressive behavior worsened, and twitching appeared in the upper limbs. Due to his aggression, the risperidone dose was further increased to $4.5 \mathrm{mg}$ daily. The patient also used valproate (600-1000 $\mathrm{mg}$ daily) for epilepsy.

After the increase in the dose of risperidone, compulsive involuntary movements, shivering, and sweating emerged. Because of these movement symptoms, first chlorprothixene and later risperidone were discontinued. The discontinuation of risperidone immediately ameliorated the movement symptoms. However, the patient's behavioral symptoms continued to fluctuate, and quetiapine (150$200 \mathrm{mg}$ daily) was initiated with no response. The movement symptoms increased again. Haloperidol $1.5 \mathrm{mg}$, clonazepam $1 \mathrm{mg}$, and diazepam $2 \mathrm{mg}$ daily were introduced to relieve the symptoms, but without response. Instead, the behavioral symptoms worsened, and the movement symptoms became gradually constant; in addition to the aforementioned movement symptoms, choreoathetoid-type movements emerged in the upper limbs. The patient was distressed and his communicative abilities deteriorated. 
Due to the worsening of the movement symptoms, the quetiapine, and temporarily also the valproate, were discontinued, but short-term levomepromazine (phenothiazine) medication was prescribed for aggressive behavior. The patient was temporarily hospitalized and piperidine $2 \mathrm{mg}$ daily was introduced. Eventually, approximately 5 months after the movement symptoms had started, all antipsychotics were discontinued. Despite the discontinuation, the serious movement symptoms continued and worsened. The patient suffered from continuous involuntary choreoathetoid movements affecting the trunk, limbs, neck, facial area, and mouth. Twitching dyskinetic extensions appeared in the back and neck area. Due to the movement symptoms, the patient had insomnia and eating problems, leading to severe weight loss (12 $\mathrm{kg}$ in 8 months). The patient was anxious and in constant pain. Diazepam relieved these symptoms temporarily. An MRI was performed for differential diagnostics, and the patient was tested for Huntington's and Wilson's diseases and neuroacanthocytosis. A final diagnosis of severe TD was made when the patient was 17 years and 7 months old.

The patient's severe movement symptoms led to self-mutilation, with several lacerations and infected wounds, resulting in sepsis and treatment in an intensive care unit. Multiple conservative treatment approaches for TD failed (incl. medical cannabis, tetrabenazine, levetiracetam, and piperidine). Finally, at the age of 19 years, the patient was referred to a neurosurgeon for evaluation for DBS, and the decision to undertake pallidal DBS was made. At the age of 19 years and 5 months, based on a 3T MRI performed under general anesthesia, electrodes were stereotactically implanted into the visually defined globus pallidus interna target (Fig. 1). DBS ameliorated the TD symptoms remarkably. Anxiety, restlessness, behavioral symptoms, and self-destructive behavior ceased, and the patient's functional capacity and communicative skills gradually recovered to the previous level. 


\section{Discussion}

Delayed diagnosis of drug-induced EPS may lead to deleterious consequences, as presented in our case. With our patient, it took too long to reach a diagnosis of TD, and even after the initial diagnosis, antipsychotics were still prescribed. There may have been several reasons for the diagnostic delay. The patient's extremely severe manifestation of movement symptoms and young age did not fit the expected picture of TD. EPS were also misinterpreted as behavioral symptoms, which possibly led to overmedication. ID and communication deficits further complicated the diagnosis.

It should be kept in mind that SGA-induced EPS, including TD, are a possibility also in children and adolescents (Carbon et al. 2015, Garcia-Amador et al. 2015, Pringsheim et al. 2017). There is not enough information available on the long-term effects of SGAs on the developing central nervous system; the studies on EPS and TD in pediatric patients cover only short time periods. Despite this, treatment periods tend to be long in clinical reality.

Patients with a diagnosis of ID do not clearly fall under any distinct medical specialty, which creates a core problem complicating the diagnosis of drug-induced adverse effects. These patients often have multifaceted health problems, and as each clinician attempts to ameliorate the symptoms, the risk of overmedication increases. To avoid serious and possibly far-reaching adverse effects related to antipsychotics, greater emphasis should be placed on the systematic follow-up of children and adolescents. Multi-professional teamwork is essential, and specialized units for the management of neuropsychiatric disorders in ID patients are needed (Bjelogrlic-Laakso et al. 2014).

If medication fails to relieve serious behavioral symptoms related to ASD and ID, other treatment approaches, such as ECT, should be considered on a case-by-case basis. DBS should be considered in cases of severe TD. 


\section{Disclosures}

K.K. has received a study grant from the Foundation for Pediatric Research and the Finnish Brain Foundation. No other conflicts of interest exist. N.B.-L. has no conflicts of interest. L.P. has received lecture fees from Biocodex, Shire Plc. and has been sponsored to travel and attend a medical congress by Biocodex, Shire PIc. K.L. has received lecture fees from Medtronic Ltd. and Abbot and has been sponsored to travel and attend a medical congress by Medtronic Ltd., Boston Scientific, and Abbot (former St. Jude Medical). K.J. has received lecture fees from Medtronic Ltd., Otsuka Pharmaceutical Ltd., and Lundbeck Ltd. and has been sponsored to travel and attend a medical congress byMedtronic Ltd.

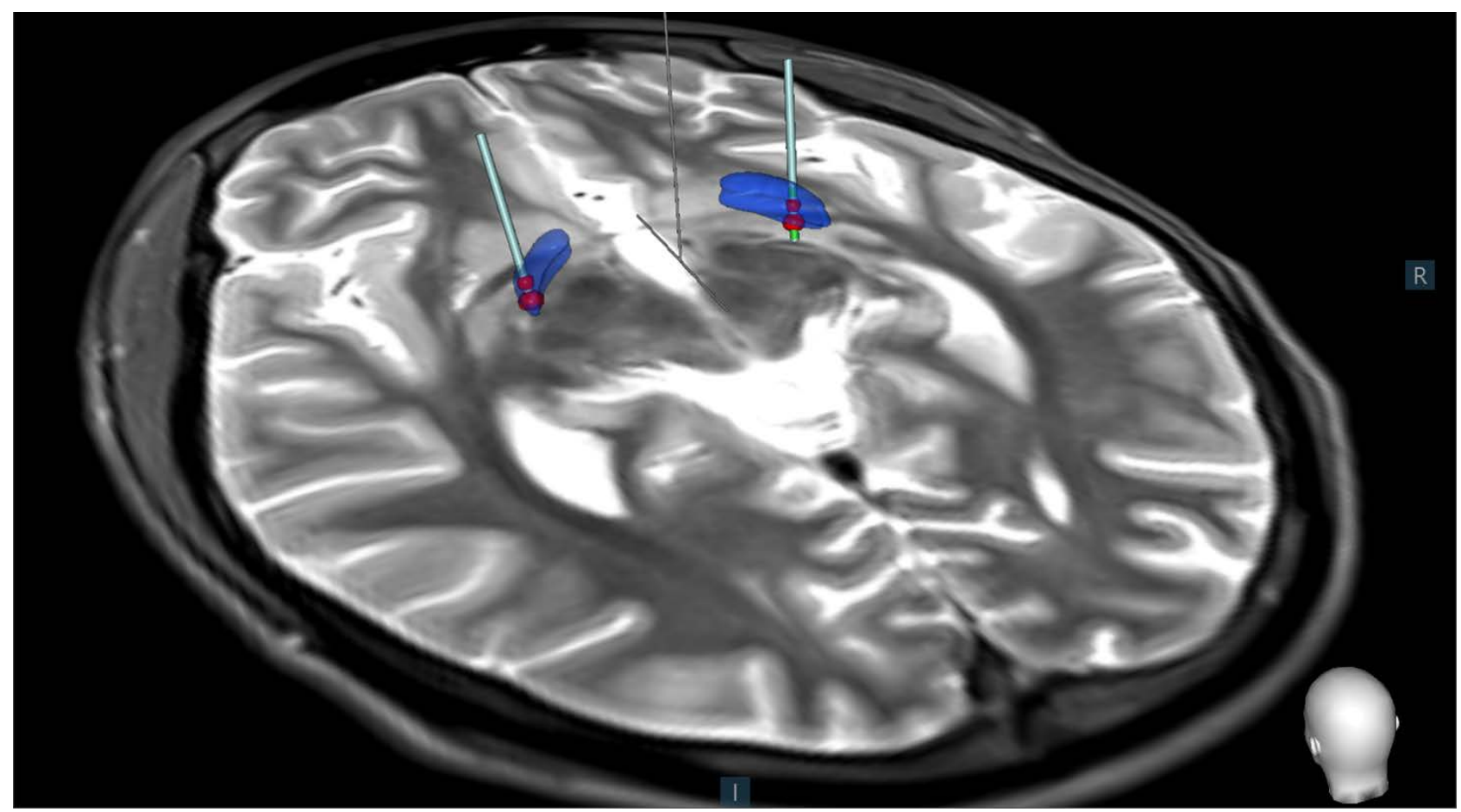

Figure 1.

Deep brain stimulation implant bilaterally in the globus pallidum interna (Gpi) is demonstrated. The borders of the Gpi (blue color) were manually identified from 3T MRI images and 3D reconstruction was generated using Medtronic Suretune 3 software (orange color). 


\section{References}

Bjelogrlic-Laakso N., Aaltonen S., Dorn T., Arvio M. Need for special units for the management of neuropsychiatric disorders in people with intellectual disabilities. Acta Psychiatr Scand 130:77-79, 2014.

Carbon M., Kapoor S., Sheridan E., Al-Jadiri A., Azzo S., Sarkaria T., Kane J.M., Saito E., Correll C.U. Neuromotor adverse effects in 342 youth during 12 weeks of naturalistic treatment with 5 second-generation antipsychotics. J Am Acad Child Adolesc Psychiatry 54:727, 2015.

Elia A.E., Bagella C.F., Ferré F. Zorzi G., Calandrella D., Romito L.M. deep brain stimulation for dystonia due to cerebral palsy: A review. Eur J Paediatr Neurol 22:308-315, 2018.

Garcia-Amador M., Merchan-Naranjo J., Tapia C., Moreno C., Castro-Fornieles J., Baeza I., De La Serna E., Alda J.A., Munoz D., Andres Nestares P., Cantarero C.M., Arango C. Neurological adverse effects of antipsychotics in children and adolescents. J Clin Psychopharmacol 35:686-693, 2015.

Lerner P.P., Miodownik C., Lerner V. Tardive dyskinesia (syndrome): Current concept and modern approaches to its management. Psychiatry Clin Neurosc 69:321-334, 2015.

Macerollo A., Deuschl G. Deep brain stimulation for tardive syndromes: Systematic review and metaanalysis. J Neurol Sci 389:55-60, 2018.

Park S.Y., Cervesi C., Galling B., Molteni S., Walyzada F., Ameis S.H., Gerhard T., Olfsson M., Corrella C.U. Antipsychotic use trends in youth with autism spectrum disorder and/or intellectual disability: A meta-analysis. J Am Acad Child Adolesc Psychiatry 55:468e4, 2016. 
Pringsheim T., Ho J., Sarna J.R., Hammer T., Patten S. Feasibility and relevance of antipsychotic safety monitoring in children with tourette syndrome: a prospective longitudinal study. J Clin Psychopharmacol 37:498-504, 2017.

Sajith S.G., Liew S.F., Tor P.C. Response to electroconvulsive therapy in patients with autism spectrum disorder and intractable challenging behaviours associated with symptoms of catatonia. J ECT 33:63-67, 2017.

Sheehan R., Horsfall L., Strydom A., Osborn D., Walters K., Hassiotis A. Movement side effects of antipsychotic drugs in adults with and without intellectual disability: UK population-based cohort study. BMJ open 7:2017406, 2017.

Wachtel L.E., Shorter E., Fink M. Electroconvulsive therapy for self-injurious behaviour in autism spectrum disorders: recognizing catatonia is key. Curr Opin Psychiatry 31:116-122, 2018. 